\title{
Traffic assignment models in road evacuation
}

\author{
M. Luisa De Maio, G. Musolino \& A. Vitetta \\ Università degli Studi Mediterranea di Reggio Calabria, \\ DIMET-Dipartimento di Informatica Matematica Elettronica e Trasporti, \\ Italy
}

\begin{abstract}
In this paper a traffic assignment model is specified in road emergency conditions. The proposed model takes into account two classes of users: some users in emergency conditions repeat the same choice made in ordinary conditions, some others make a different choice. The basic concept for the proposed model is derived from day-to-day models. The path choice model, which is inside the traffic assignment model, has to take into account three types of information which influence users' choices: historical information related to network experience in ordinary conditions, real time information related to the current emergency situation, and users' forecasting of the network evolution based on current experience. A non-equilibrium dynamic model is adopted in order to simulate path flows on the network. A review of static and dynamic traffic assignment models in emergency conditions is reported.

Keywords: traffic assignment, dynamic traffic assignment, dynamic equilibrium, day-to-day models, non-equilibrium models.
\end{abstract}

\section{Introduction}

A traffic assignment (TA) model simulates how travel demand and transport networks interact in transportation systems. These models allow the calculation of performances (costs) and vehicular flows on each element (link) of the transport network, resulting from origin-destination demand flows, users' path choice behaviour and link costs.

TA models may be classified according to different criteria. The first one concerns the dependence of link cost variables on vehicular flows. Fixed costs or uncongested networks result in non-equilibrium traffic assignment models. Variable costs or congested networks lead to equilibrium traffic assignment 
models. Equilibrium consists of finding path (link) flows mutually consistent with the corresponding path (link) costs. This problem can be formulated with fixed-point models, variational inequalities or optimization models [1].

Two kinds of equilibrium traffic assignment models are present in the literature: User Equilibrium and System Optimimum. The former consists of finding path flows (First Wardrop principle), in order for a user to minimize their individual costs. On the other hand, the latter consists of finding path flow-path cost consistency (Second Wardrop principle), in order to achieve the minimum total cost on the network, although some users may follow a non-minimum cost path [2].

The second criterion allows the classification of Traffic Assignment models into Static (STA) and Dynamic (DTA) models. STA models simulate a transport system in stationary conditions, when travel demand, path choices and the transport network are consistent in a reference time. DTA models remove stationary assumptions, allowing transport system evolution to be represented as in the case of emergency conditions when travel demand peaks, temporary link and node capacity variations, queue formation and dispersion occur. Therefore STA models achieve reasonable results when the time horizon simulation is long enough to assume stationary conditions. On the contrary, when frequent changes in network features and demand occur, like in emergency conditions, the stationary hypothesis cannot be assumed.

In the last few years, researchers and authorities have focused attention on transportation system simulations in emergency conditions. Therefore many issues have been treated and several works are available in the literature. An overview of the risk evaluation and general methodology applied in road evacuation can be found in $[3,4]$. In order to model a transportation system, the travel demand system, transport supply system and their interactions have to be simulated: for travel demand simulations in emergency conditions please refer to [5-9]; for transport supply simulations and traffic assignment in emergency conditions please refer to $[10,11]$. Dynamic routing of emergency vehicles is treated in $[12,13]$. Evacuation planning methods and tools available in the literature were analysed in [14-18].

In this paper traffic assignment in emergency conditions is treated. A dynamic model is given which allows the calculation of path flows related to each origindestination o-d pair in emergency conditions, taking into account both the behaviour of users who reconsider the choice made in ordinary conditions and users who do not reconsider the choice made in ordinary conditions. The main attention is focused on a path choice model in emergency conditions. Path flows related to each o-d pair in emergency conditions are then simulated through a dynamic non-equilibrium model.

After this introduction, Section 2 reports a general view of static and dynamic models applied in emergency conditions; Section 3 describe a dynamic algorithm; in Section 4 the proposed model is specified; in Section 5 the nonequilibrium model is described; final remarks are reported in Section 6. 


\section{Static versus dynamic models in emergency conditions}

In general, traffic assignment models consist of three groups of models: the demand model, supply model and demand-supply interaction model. Demand models may be monoclass or multiclass and, in relation to the time in which route choice is made, they may be pre-trip, en-route or hybrid. Supply models may be macroscopic, mesoscopic and microscopic, and information to users may be available (historical or real time) or unavailable. Demand-supply interaction may be non-equilibrium like Network Loading (NL), equilibrium like System Optimum (SO) or User Equilibrium (UE) or Boundedly Rational (BR).

In the following we are going to propose a classification of STA and DTA models (see Figure 1): the time in which route choice is made (demand model) and assignment adopted approach (demand-supply interaction model) are considered. The STA models may be non-equilibrium or equilibrium. For the stationary hypothesis, travel time remains unchanged: therefore users' choices are completely pre-trip. Similarly, DTA models may be non-equilibrium or equilibrium. In emergency conditions the stationary hypothesis is eliminated: new concepts of instantaneous travel time and experienced travel time have to be introduced. Route travel time is obtained summing travel times of links which belong to the considered route. The instantaneous travel time is calculated considering the links travel time at the origin departure time. The experienced travel time is calculated considering links travel time at the moment in which the single link is approached.

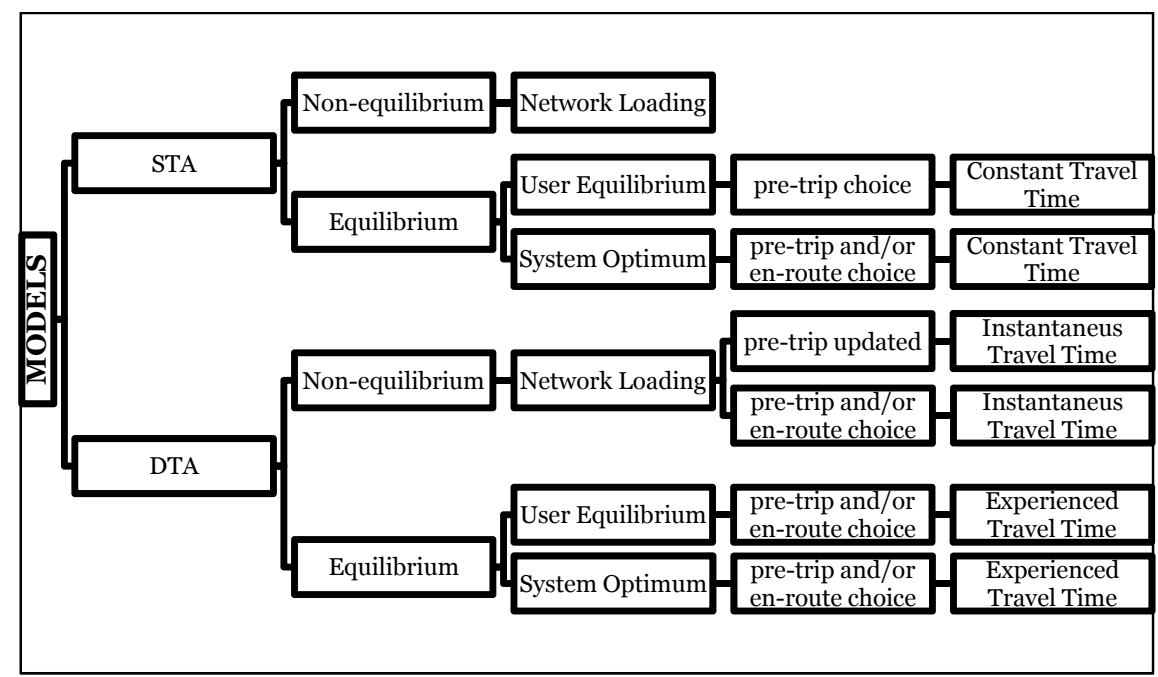

Figure 1: Traffic assignment models classification.

First, non-equilibrium DTA models are treated and instantaneous travel time is considered. Users might choose pre-trip, periodically updating travel time: shortest routes are regularly updated considering current traffic conditions and 
assignment is repeated considering new vehicles which are beginning the trip [19]. Otherwise, users might choose using a hybrid approach: choice is made pre trip and modified en route in relation to actual network conditions. Therefore travellers are allowed to abandon the current chosen route for a better one; because of link travel times changes occurred after the previous route choice.

Second, equilibrium DTA models are explained. The steady conditions hypothesis is no longer valid: therefore the equilibrium concept has to be formulated in a different way. Users make route choice based on currently experienced times on the network, which depend on the departure time. Hence, users experience the same travel time on the network only if they are travelling between the same o-d pair and left at the same departure time [19].

In addition to this, another concept of dynamic equilibrium is available in the literature. In ordinary conditions it is reasonable to assume that users have an exact travel time perception and base choices on their experience of the network: when users have a certain experience of the network, equilibrium is achieved and all users experience the same travel time.

Emergency conditions rarely occur and therefore users have no experience of the network. Hence, equilibrium, as defined in ordinary conditions, cannot be achieved. A perceived utility has to be specified to introduce the equilibrium concept in emergency conditions. Attributes used for utility specification may be: travel time, departure time, network reliability, forecasted conditions, early/late penalty and so on. It follows that the Wardrop equilibrium concept can be re-written in the following way: "the perceived utilities of all routes actually used are equal and less than those which would be experienced by a single vehicle on any unused route". In other words, dynamic equilibrium is achieved when the perceived utility is the same for all users, but the travel time is not necessarily the same. In detail, in [20], the perceived utility is called the payoff and the dynamic equilibrium is stated as follows: "A dynamic equilibrium solution is the set of departure rates over a time interval $\mathrm{T}$ that a) provides all identical commuters the same payoff; and, b) has a utility greater than the utility obtained by departing at a time outside the interval $\mathrm{T}$ (not necessarily contiguous)".

\section{A dynamic algorithm}

The solution of the dynamic equilibrium is obtained by an iterative process, characterized by a non-definite number of steps. As a consequence "DTA models usually find and approximate equilibrium which sufficiently converges to true equilibrium and that is obtainable in a reasonable amount of time" [19]. As stated in [19], the features of a DTA solution can be summed up as follows:

- vehicles departing at different times are assigned with different routes;

- vehicles departing at the same departure time between the same o-d pair but taking different routes should have the same experienced travel time;

- experienced travel time cannot be realized at departure, but only at the end of the trip. 
According to [19], three algorithms have to be used iteratively in order to find an equilibrium solution:

- network loading, which calculates route travel times;

- $\quad$ path set update which calculates the shortest paths;

- path assignment adjustment, which assigns vehicles to routes in order to approximate the dynamic equilibrium.

After performing Path Assignment Adjustment, the algorithm returns to the route evaluation step in order to determine the traffic pattern that would result from the new route choices (route flows). Thus, the three steps work in a sequential way: the output of network loading provides the input for path set update; the output of path set update provides the input for path assignment adjustment; and the output of path assignment adjustment provides the input for network loading. These three steps are repeated until a stopping criterion is met.

\section{Dynamic proposed model}

Dynamic models are introduced in order to take into account changes in demand and supply, which usually occur during emergency situations. Dynamic equilibrium concepts available in the literature are explained in Section 2. One of the main issues to be investigated is related with users' experience of the network. On the one hand, because of users' lack of experience on the network in emergency conditions, equilibrium, as stated in static conditions, cannot be achieved. On the other hand, users' choices are influenced by experience of the network in ordinary conditions.

In the proposed model, whose basic concept is inspired by day-to-day dynamic models [21-26], influence of users' experience of the network on users' choices is taken into account. The proposed model allows the calculation of path flows related to each o-d pair, $\mathrm{h}_{\mathrm{od}}{ }^{\mathrm{t}}$, considering two different components. The proposed model can be specified as follows:

$$
\mathrm{h}_{\mathrm{od}}{ }^{\mathrm{t}}=\alpha \cdot \mathrm{d}_{\mathrm{od}} \cdot \mathrm{p}_{\mathrm{od}}{ }^{\mathrm{t}} \cdot\left(\mathrm{V}^{\mathrm{t}}\right)+(1-\alpha) \cdot \mathrm{h}_{\mathrm{od}}^{\mathrm{t}-1}
$$

In the following the two components which compose the model, variables and parameters are explained. It is worth noting that variables with $t$ as superscript refer to current emergency conditions, whereas those with $t-1$ as superscript refer to the previous day ordinary conditions. The first component describes the behaviour of users who reconsider the choice made in ordinary conditions, in relation to actual emergency network conditions. This component depends on:

- $\quad \alpha$, the probability to reconsider the choice made in ordinary conditions;

- $\quad \mathrm{d}_{\text {od }}$, the travel demand on the considered o-d pair;

- $\mathrm{p}_{\mathrm{od}}{ }^{\mathrm{t}}$, the probability to choose a path different to the one chosen in ordinary conditions.

The probability to choose an alternative path, $\mathrm{p}_{\mathrm{od}}$, depends on the systematic utility, $\mathrm{V}^{\mathrm{t}}$, calculated considering both the actual path costs $\mathrm{g}_{\mathrm{k}}{ }^{\mathrm{t}}$, evaluated in emergency conditions, and the expected systematic utility on the previous day $\mathrm{V}^{\mathrm{t}-1}$, based on ordinary conditions network experience. These two factors, $\mathrm{g}_{\mathrm{k}}{ }^{\mathrm{t}}$ and 
$\mathrm{V}^{\mathrm{t}-1}$, have a different weight on the $\mathrm{V}^{\mathrm{t}}$ calculation, provided through the $\beta$ and 1$\beta$ values. A possible formulation is:

$$
\mathrm{V}^{\mathrm{t}}=\beta \cdot \mathrm{g}_{\mathrm{k}}{ }^{\mathrm{t}}+(1-\beta) \cdot \mathrm{V}^{\mathrm{t}-1}
$$

In its turn, $\mathrm{V}^{\mathrm{t}-1}$ is calculated using (2): therefore it takes into account experience related to ordinary conditions on the $\mathrm{t}-2$ day. In this way the entire past experience is included in the proposed model.

The second component of the model describes the behaviour of users who, in emergency conditions, do not reconsider the choice made in ordinary conditions. Therefore there are a number of users who will repeat the same choice they are used to making in ordinary conditions. This number is obtained by multiplying:

- $(1-\alpha)$, the probability to not reconsider the choice made in ordinary conditions, and

- $\mathrm{h}_{\mathrm{od}}{ }^{\mathrm{t}-1}$, the path flow on the considered o-d pair in ordinary conditions on the $\mathrm{t}-1$ day.

The values of $\alpha$ and $\beta$ have to be calibrated.

After describing the general framework of the proposed model, we are going to focus our attention on the path choice model in emergency conditions $\left(\mathrm{p}_{\mathrm{od}}{ }^{\mathrm{t}}\right)$, which allows the calculation of the probability to choose a path different to the one chosen in ordinary conditions. The emergency conditions path choice model specification must take into account three different elements which influence users' choices: first, previous experience, second, real time information, and third, forecasting based on current experience of the network. These three elements are introduced through attributes used for model specification.

Another issue to be investigated in relation to the emergency conditions path choice model specification is related to the mathematical formulation of the model. It might be the same both in ordinary and emergency conditions: perceived attribute values in emergency conditions are different to the ones in ordinary conditions. On the other hand, an alternative mathematical formulation for the emergency conditions path choice model specification might be assumed, in order to better describe users' behaviour in non-ordinary conditions.

In general, a path choice model, as for every demand model, should be specified on three levels: generation of alternatives (Generation), perception of choice set/s (Perception), and choice of alternatives into the choice set (Choice). Several researchers have recently dealt with this issue: the probability that a generic user chooses route $\mathrm{k}$ is calculated in two steps in [27]. In the first step the choice set is generated (Generation) whereas, in the second step, the choice is simulated. When $\mathrm{F}$ is a set which contains all feasible routes for a generic user and $\mathrm{S}$ is a set which contains all feasible sub-sets I, extracted from $\mathrm{F}$, the probability that the alternative $\mathrm{k}$ is chosen depends on the probability that a generic user chooses sub-set I (Perception) to which k belongs. Manski [27] proposes to calculate the probability that a generic user chooses route $\mathrm{k}$ by summing, for all $\mathrm{I}$ belonging to $\mathrm{S}$, the product between the probability that a generic user chooses sub-set I, considering I not empty, and the probability that a generic user chooses alternative $\mathrm{k}$, related to the choice of sub-set I (Choice). It is 
worth noting that the specification of the three levels explained above is not always needed, because alternatives are sometimes automatically identified. The traffic zones, in which the study area is divided, are the alternatives for the distribution model. Anyway, at the path choice dimension, a considerable amount of alternatives can be found. So specifying the model throughout three levels is needed, in order to simulate correctly the perception process. Several factors make the simulation of the route perception process more complicated [28-30]: a non-deterministic problem nature, tastes variability caused by different individual experiences, a considerable amount of alternatives for each (origin, destination) pair and their overlapping, users' compliance with information given by the Advanced Traveller Information System (ATIS) [3133]).

\section{A dynamic non-equilibrium model formulation}

The output obtained from the model proposed in the previous section is $\mathrm{h}_{\mathrm{od}}{ }^{\mathrm{t}}$. In this section the way $\mathrm{h}_{\mathrm{od}}{ }^{\mathrm{t}}$ may be simulated is reported.

Given an origin-destination pair (od), the generalized cost of path $k, g_{k}{ }^{t}$, is assumed to be equal to the path travel time. In this section we are going to focus our attention on $t$, which represents the current emergency conditions: for this reason now superscript $t$ will be omitted.

Preliminarily, it is necessary to introduce some concepts about time:

- $\mathrm{t}_{0}$ is the initial time instant;

- $\tau$ is the decision time interval of users at the origin (pre-trip choice);

- $\tau^{*}$ is the reference time instant of decision time interval $\tau$ (departure times of all users in $\tau$ are concentrated in $\tau^{*}:$ it could be the left-bound, the right-bound or the central value).

The travel demand flow on the origin-destination (od) pair, $\mathrm{d}_{(\mathrm{od})}$, and the choice probability of path $\mathrm{k}, \mathrm{p}_{\text {(od)k }}$ (now referred to as $\mathrm{p}_{\mathrm{k}}$ for simplicity's sake) change with the time instant $\tau^{*}$. In particular the choice probability of path $\mathrm{k}$ at time instant $\tau^{*}, \mathrm{p}_{\mathrm{k}, \tau *}$, depends on the path cost, $\mathrm{g}_{\mathrm{k}, \tau^{*}}$, forecasted by the user before departing from the origin.

At time instant $\tau^{*}$, the pre-trip choice made by the user, who does not know the current transport system conditions, is determined by his/her knowledge of historical costs experienced in the past. The forecasted costs can be estimated through a cost updating model.

At equilibrium the transport system is permanently in ordinary conditions so it can be assumed that costs forecasted by users coincide with the exact current ones [1]. In emergency conditions the assumptions of equilibrium existence do not hold because path costs change as a consequence of transport system modifications.

Cost modifications are due to:

- travel demand modifications, as regards variations of emission, distribution and mode choice dimensions; 
- transport supply modifications, due to interventions implemented by the police and/or the damages produced by events occurring on the transport system.

Supply modifications can influence user path choice. However, the user still chooses the path also on the basis of his/her historical information even if there is a high probability that historical costs (in ordinary conditions) differ from current costs (in emergency conditions). In addition to the historical information, the user takes into account the current network situation and real time information, if available.

The path flow related to the considered o-d pair and obtained as the output of the model proposed in the previous section $\left(\mathrm{h}_{\mathrm{od}}{ }^{\mathrm{t}}\right)$ may be thought of as the sum of travel demand flow on paths $\mathrm{k}$ used for trips from the origin o to the destination d. Therefore, for each decision time instant, $\tau^{*}$, the travel demand flow on path $\mathrm{k}$, $\mathrm{h}_{\mathrm{k}, \tau *}$, is given by the product between $\mathrm{d}_{(\mathrm{od}), \tau}$ and $\mathrm{p}_{\mathrm{k}, \tau *}$ :

$$
\mathrm{h}_{\mathrm{k}, \tau *}=\mathrm{p}_{\mathrm{k}, \tau *} \mathrm{~d}_{(\mathrm{od}), \tau}
$$

where

- $\mathrm{d}_{(\mathrm{od}) \tau}=\int_{\tau} z_{\text {(od) }}(\mathrm{t}) \mathrm{dt}$ is the travel demand flow of the origin-destination (od) pair at decision time interval $\tau$;

- $z_{(\mathrm{od})}(\mathrm{t})$ is the time-dependent demand function for the (od) pair at generic time instant $\mathrm{t}$.

In relation to $\mathrm{d}_{(\mathrm{od}), \tau}$, refer to [7]: the demand analysis for the transportation system simulation in emergency conditions is treated. Specification and calibration of models is presented and data achieved from both SP (Stated Preference) and RP (Revealed Preference) surveys are used [5].

The travel demand flow $h_{k, \tau *}$ along path $\mathrm{k}$ departing at time instant $\tau^{*}$ is distributed at a subsequent instant $\mathrm{t}\left(\mathrm{t} \geq \tau^{*}\right)$ on the links belonging to path $\mathrm{k}$. It is possible to determine the probability $q_{\mathrm{a}}^{\mathrm{k}}\left(\mathrm{t} \mid \tau^{*}\right)$ of being on link a belonging to path $\mathrm{k}$ at the time instant $\mathrm{t}$ conditional upon the decision (to choose path $\mathrm{k}$ ) at time instant $\tau^{*}$.

The probability $q_{\mathrm{a}}^{\mathrm{k}}\left(\mathrm{t} \mid \tau^{*}\right)$ depends on traffic conditions or on the evolution of costs on links belonging to path $\mathrm{k}$ from $\tau^{*}$ to $\mathrm{t}\left(\mathrm{t} \geq \tau^{*}\right)$.

The sum of probabilities $q_{\mathrm{a}}{ }^{\mathrm{k}}\left(\mathrm{t} \mid \tau^{*}\right)$ for all links belonging to path $\mathrm{k}$, calculated at time instant $t$ and for the same time instant $\tau^{*}$, is equal to one:

$$
\Sigma_{\mathrm{a} \in \mathrm{k}} q_{\mathrm{a}}^{\mathrm{k}}\left(\mathrm{t} \mid \tau^{*}\right)=1 \forall \mathrm{k}, \mathrm{t}, \tau^{*}\left(\mathrm{t} \geq \tau^{*}\right)
$$

Traffic flow on link a at time instant $t$ is given by the sum, for all origindestination pairs (od), for all paths containing the link a and for all decision time instants $\tau^{*}$ belonging to the $\left[\mathrm{t}_{0}, \mathrm{t}\right]$ interval, of the products $q_{\mathrm{a}}{ }^{\mathrm{k}}\left(\mathrm{t} \mid \tau^{*}\right) \mathrm{h}_{\mathrm{k}, \tau^{*}}$ :

$$
f_{a}(\mathrm{t})=\Sigma_{(\mathrm{rs})} \Sigma_{\mathrm{k}: \mathrm{a} \in \mathrm{k}} \Sigma_{\tau *=[\mathrm{t} 0, \mathrm{t}]} q_{\mathrm{a}}^{\mathrm{k}}\left(\mathrm{t} \mid \tau^{*}\right) \mathrm{h}_{\mathrm{k}, \tau *}
$$




\section{Conclusions}

In this paper an analysis of static and dynamic models applied in emergency conditions was reported. A dynamic algorithm is described in order to find a dynamic equilibrium solution. In addition to this, a model is proposed in order to calculate path flows on each o-d pair in emergency conditions: both the behaviour of users who change their choices in emergency conditions and users who repeat ordinary conditions choices is simulated. A non-equilibrium model provides simulation of path flows obtained by the previous step.

Future developments may be related to further specification of the proposed model and experimentation on a real network.

\section{Acknowledgement}

Partially supported by national MIUR under PRIN2009 grant no. 2009EP3S42 001.

\section{References}

[1] Cascetta E., Transportation Systems engineering: theory and methods, Kluwer Academic Publisher, 2001.

[2] Wardrop J.G., Some theoretical aspects of road traffic research, Proceedings, Institution of Civil Engineers, Part II 1, pp. 325-378, 1952.

[3] Russo F. and Vitetta A., Risk evaluation in a transportation system. International Journal of Sustainable Development and Planning, 1 (2), pp. 170-191, 2006.

[4] Russo F. and Vitetta A., Safety of users in road evacuation: General methodology and main results. WIT Transactions on the Built Environment, Volume 96, Urban Transport XIII, Urban Transport and the Environment in the 21st century, pp. 763-772, 2007.

[5] Russo F. and Chilà G., Safety of users in road evacuation: RP vs. SP surveys in demand analysis. WIT Transactions on the Built Environment, Volume 101, Urban Transport XIV, Urban Transport and the Environment in the 21st century, Brebbia C. A. (ed.), WIT Press, Southampton, pp. 703713, 2008.

[6] Russo F. and Chilà G., Safety of users in road evacuation: Modelling and DSS for demand. WIT Transactions on Ecology and the Environment, Volume 120, Sustainable Development and Planning IV, vol.1, Brebbia C. A. (ed.), WIT Press, Southampton, pp. 465-474, 2009.

[7] Russo F. and Chilà G., Safety of users in road evacuation: demand models. WIT Transactions on the Built Environment, Volume 96, Urban Transport XIII, Urban Transport and the Environment in the 21st century, Brebbia C. A. (ed.), WIT Press, Southampton, pp. 773-782, 2007.

[8] Russo F. and Chilà G., Demand models in road evacuation: a synopsis of recent contributions. WIT Transactions on the Built Environment, Volume 116, Urban Transport XVII, Urban Transport and the Environment in $21^{\text {st }}$ 
Century, Pratelli and Brebbia C. A. (ed.), WIT Press, Southampton, pp. 601-614, 2011.

[9] Russo F. and Chilà G., A statistical approach to analyse user behaviour in road evacuation. WIT Transactions on the Built Environment, Volume 117, Safety and Security engineering IV, Guarascio M., Reiners G. Brebbia C. A. and Garzia F. (ed.), WIT Press, Southampton, pp. 377-390, 2011.

[10] Musolino G. and Vitetta A., Short-term forecasting in road evacuation: calibration of a travel time function. In: Brebbia C. A. Pratelli, A. Eds, Urban Transport and the Environment in the 21st century, WITPress, Southampton (GBR), 2011, pp. 615-626, 2011.

[11] Marcianò F.A., Musolino G. and Vitetta A., Within day traffic assignment and signal setting in road evacuation: a procedure with explicit path enumeration. WIT Transactions on the Built Environment, Volume 117, Safety and Security engineering IV, Guarascio M., Reiners G. Brebbia C. A. and Garzia F. (ed.), WIT Press, Southampton, pp. 403-414, 2011.

[12] Polimeni A. and Vitetta A., Dynamic vehicle routing in road evacuation: a model for route design. WIT Transactions on the Built Environment, Volume 116, Urban Transport XVII, Urban Transport and the Environment in $21^{\text {st }}$ Century, Pratelli and Brebbia C. A. (ed.), WIT Press, Southampton, pp. 627-638, 2011.

[13] Polimeni A. and Vitetta A., Dynamic vehicle routing in road evacuation: route design experimentation. WIT Transactions on the Built Environment, Volume 117, Safety and Security engineering IV, Guarascio M., Reiners G. Brebbia C. A. and Garzia F. (ed.), WIT Press, Southampton, pp. 391-402, 2011.

[14] Russo F. and Rindone C., Safety of users in road evacuation: Modelling and DSS for LFA in the planning process. WIT Transactions on Ecology and the Environment, Volume 120, Sustainable Development and Planning IV, vol.1, Brebbia C. A. (ed.), WIT Press, Southampton, pp. 453-464, 2009.

[15] Russo F. and Rindone C., Safety of users in road evacuation: the logical framework approach in evacuation planning. WIT Transactions on the Built Environment, Volume 101, Urban Transport XIV, Urban Transport and the Environment in the 21st century, Brebbia C. A. (ed.), WIT Press, Southampton, pp. 751-760, 2008.

[16] Russo F. and Rindone C., Safety of users in road evacuation: planning internal processes and guidelines. WIT Transactions on the Built Environment, Volume 96, Urban Transport XIII, Urban Transport and the Environment in the 21st century, Brebbia C. A. (ed.), WIT Press, Southampton, pp. 825-834, 2007.

[17] Russo, F. and Rindone C., Planning in road evacuation: classification of exogenous activities. WIT Transactions on the Built Environment, Volume 116, Urban Transport XVII, Urban Transport and the Environment in $21^{\text {st }}$ Century, Pratelli and Brebbia C. A. (ed.), WIT Press, Southampton, pp. 639-651, 2011.

[18] Russo, F. and Rindone C., The planning process and logical framework approach in road evacuation: a coherent vision. WIT Transactions on the 
Built Environment, Volume 117, Safety and Security engineering IV, Guarascio M., Reiners G. Brebbia C. A. and Garzia F. (ed.), WIT Press, Southampton, pp. 415-425, 2011.

[19] Chiu Y.C., Bottom J., Mahut M., Paz A., Balakrishna R., Waller T. and Hicks J., A primer for Dynamic Traffic Assignment. Transportation Research Board, 2010

[20] Ramadurai, G., Ukkusuri, S. V., Zhao, J, and Pang, J. S., Linear Complementary Formulation for the Multi-user Class Single Bottleneck Problem, Transportation Research Part B: Methodological . Vol. 44(2), pp. 193-214, 2010.

[21] Daganzo C.F. and Sheffi Y., On stochastic models of traffic assignment, Transportation Science, vol. 11 (5), pp. 253-274, 1977.

[22] Horowitz J.L., The stability of stochastic equilibrium in a two link transportation network, Transportation. Research, vol. 18B (1), pp. 13-28, 1984.

[23] Davis, N. and Nihan N. L., Large Population Approximations of a General Stochastic Traffic Assignment Model, Operations Research, 1993.

[24] Watling D., Stability of the stochastic equilibrium assignment problem: a dynamical systems approach, Transportation. Research. 33B, 281-312, 1999.

[25] Watling D. and Hazelton M.L., The Dynamics and Equilibria of Day-toDay Assignment Models, Network and Spatial Economics, 3, 349-370, 2003.

[26] Cantarella G.E. and Cascetta E., Dynamic Processes and Equilibrium in Transportation Networks, Transportation Science vol. 29, pp. 305-329, 1995.

[27] Manski C., The structure of random utility models, Theory and Decision 8, (1977), pp. 229-254, 1977.

[28] Florian M. and Fox B., On the probabilistic origin of Dial's multipath traffic assignment models, Transportation Research 10 (5,) pp. 339-341, 1976.

[29] Russo F., Fuzzy Theory in Transportation Field: Fuzzy Sets for Simulating Path Choice Behaviour, In Advanced in Intelligent Systems, Morabito F.C. (ed.), IOS Press, 1997.

[30] Ridwan M., Fuzzy preference based traffic assignment problem. Transportation Research Part C 12, pp. 209-233, 1994.

[31] Golledge R., Dynamics and ITS: behavioural responses to information available from ATIS (Resource Paper), Paper presented at the IATBR 8th Meeting: Challenges and Opportunities in Travel Behaviour Research and Applications, The University of Texas at Austin, 1997.

[32] Adler J.L., Investigating the learning effects of route guidance and traffic advisories on route choice behaviour, Transportation Research Part C 9, pp. 1-14, 2001.

[33] Bifulco G. N., Simonelli F. and Di Pace R., The Role of the Uncertainty, in ATIS Applications. E. Avineri et al. (Eds.): Applications of Soft Computing, ASC 52, pp. 230-239, 2008. 